\title{
Global Regularity of the 3D Axi-symmetric Navier-Stokes Equations with Anisotropic Data
}

\author{
Thomas Y. Hou* Zhen Lei $^{\dagger} \quad$ Congming Li ${ }^{\ddagger}$
}

January 24, 2009

\begin{abstract}
In this paper, we study the 3D axisymmetric Navier-Stokes Equations with swirl. We prove the global regularity of the 3D Navier-Stokes equations for a family of large anisotropic initial data. Moreover, we obtain a global bound of the solution in terms of its initial data in some $L^{p}$ norm. Our results also reveal some interesting dynamic growth behavior of the solution due to the interaction between the angular velocity and the angular vorticity fields.
\end{abstract}

\section{Introduction.}

Despite a great deal of effort by many mathematicians and physicists, the question of whether the solution of the 3D Navier-Stokes equations can develop a finite time singularity from a smooth initial condition with finite energy remains one of the most outstanding open problems [6]. A main difficulty in obtaining the global regularity of the 3D Navier-Stokes equations is due to the presence of the vortex stretching, which is absent for the 2D problem. So far, the global regularity of the 3D Navier-Stokes equations has been obtained only for initial data which are small in some scaling invariant norm [16, 27, 19]. But the analysis for small initial data does not generalize to the 3D Navier-Stokes with large data. A more refined analysis which takes into account the special nature of the nonlinearities and the anisotropic nature of the solution near the region of blow-up seems to be needed.

In this paper, we study the global regularity of the axisymmetric Navier-Stokes equations with large initial data that have anisotropic scaling. Let $u^{\theta}$ and $\omega^{\theta}$ be the angular velocity and vorticity components of the 3D axisymmetric Navier-Stokes equations. We consider initial data for $u^{\theta}$ and $\omega^{\theta}$ that have the following scaling property:

$$
u^{\theta}(r, z, 0)=\frac{1}{\epsilon^{1-\delta}} U_{0}(\epsilon r, z), \quad \omega^{\theta}(r, z, 0)=\frac{1}{\epsilon^{1-\delta}} W_{0}(\epsilon r, z)
$$

*Applied and Comput. Math, Caltech, Pasadena, CA 91125. Email: hou@acm.caltech.edu.

${ }^{\dagger}$ Applied and Comput. Math, Caltech, Pasadena, CA 91125. Email: zhenlei@acm.caltech.edu.

${ }^{\ddagger}$ Department of Applied Mathematics, University of Colorado, Boulder, CO. 80309. Email: cli@colorado.edu 
where $r=\sqrt{x^{2}+y^{2}}, \delta$ and $\epsilon$ are some small positive parameters, and the rescaled profiles $U_{0}$ and $W_{0}$ are bounded in $L^{2 p}$ and $L^{2 q}$ respectively for some $p$ and $q$ with $p=2 q$. We note that these initial data are not small. In fact, we have

$$
\left\|\mathbf{u}_{0}\right\|_{L^{2}\left(\mathbb{R}^{2} \times[0,1]\right)}\left\|\nabla \mathbf{u}_{0}\right\|_{L^{2}\left(\mathbb{R}^{2} \times[0,1]\right)}=\frac{C_{0}}{\epsilon^{4-2 \delta}} \gg 1
$$

for $\epsilon$ small, where $\mathbf{u}_{0}$ is the initial velocity vector (we use bold letters to denote vector fields throughout this paper). Thus the classical regularity analysis for small initial data does not apply to these sets of anisotropic initial data.

In this paper, we prove the global regularity of the 3D axisymmetric Navier-Stokes equations for both initial data given by (1) and (2) by exploring the anisotropic structure of the solution for $\epsilon$ small. We also obtain a global bound on $\left\|u^{\theta}\right\|_{L^{2 p}}$ and $\left\|\omega^{\theta}\right\|_{L^{2 q}}$ in terms of their initial data. Note that by using the scaling invariance property of the Navier-Stokes equations, our global regularity result also applies to the following rescaled initial data

$$
u^{\theta}(r, z, 0)=\frac{1}{\epsilon^{2-\delta}} U_{0}\left(r, \frac{z}{\epsilon}\right), \quad \omega^{\theta}(r, z, 0)=\frac{1}{\epsilon^{3-\delta}} W_{0}\left(r, \frac{z}{\epsilon}\right)
$$

and

$$
u^{\theta}(r, z, 0)=\frac{1}{\epsilon} U_{0}\left(\frac{r}{\epsilon^{1-\delta}}, \frac{z}{\epsilon}\right), \quad \omega^{\theta}(r, z, 0)=\frac{1}{\epsilon^{2}} W_{0}\left(\frac{r}{\epsilon^{1-\delta}}, \frac{z}{\epsilon}\right) .
$$

For the rescaled initial data (2) and (3), our analysis suggests that $\left\|u^{\theta}\right\|_{L^{2 p}}$ may experience a rapid growth dynamically due to the contribution from $\omega_{0}^{\theta}$. On the other hand, the growth of $\left\|\omega^{\theta}\right\|_{L^{2 q}}$ is more moderate. See Remark 2 and (32)-(33) in Section 3 for more discussions. This phenomenon is similar to the solution behavior of the 1D model derived by Hou-Li in [9] where they show that $\omega^{\theta}$ is essentially bounded by its initial data while $u^{\theta}$ can experience large growth dynamically due to the contribution from $\left(u_{0}^{\theta}\right)_{z}$ and $\omega_{0}^{\theta}$.

Note that the parameters $\epsilon$ in the initial data (1)-(2) and $\delta$ in (3) measure the degree of anisotropy of the initial data. If $\delta=0$, then the initial data (3) become isotropic, i.e.

$$
\mathbf{u}_{0}(x, y, z)=\frac{1}{\epsilon} \mathbf{U}_{0}\left(\frac{x}{\epsilon}, \frac{y}{\epsilon}, \frac{z}{\epsilon}\right) .
$$

Our analysis breaks down when there is no anisotropic scaling in the initial data, i.e. $\delta=0$. Clearly, if the analysis could be extended to the case of $\delta=0$, one would prove the global regularity of the 3D axisymmetric Navier-Stokes equations for general initial data by using the scaling invariance property of the Navier-Stokes equations. It is interesting to note that by using an anisotropic scaling of the initial data, we turn the global regularity of the 3D Navier-Stokes equations into a critical case of $\delta=0$.

We would like to emphasize that our global regularity results are obtained on a regular size domain, $\mathbb{R}^{2} \times[0,1]$, for initial data (1). In this sense, our results are different from those global regularity results obtained for a thin domain, $\Omega_{\epsilon}=Q_{1} \times[0, \epsilon]$ with $Q_{1}$ being a bounded domain in $\mathbb{R}^{2}$. We remark that the global regularity of the 3D Navier-Stokes equations in a thin domain of the form $\Omega_{\epsilon}$ has been studied by Raugel and Sell in a series of 
papers $[23,24,25]$. They prove the global regularity of the 3D Navier-Stokes equations under the assumption that $\left\|\nabla \mathbf{u}_{0}\right\|_{L^{2}\left(\Omega_{\epsilon}\right)}^{2} \leq C_{0} \ln \frac{1}{\epsilon}$. This is an improvement over the classical global regularity result for small data, which requires $\left\|\nabla \mathbf{u}_{0}\right\|_{L^{2}\left(\Omega_{\epsilon}\right)}^{2} \leq C^{*} \epsilon[23]$. We may interpret our global regularity result with initial data (2) as a result on a generalized thin domain. Note that the initial data given by (2) satisfy the following bound: $\left\|\nabla \mathbf{u}_{0}\right\|_{L^{2}\left(\Omega_{\epsilon}\right)}^{2}=C_{0} \epsilon^{-5+2 \delta}$ (here $\delta>0$ can be made arbitrarily small), which is much larger than the corresponding bound $C_{0} \ln \frac{1}{\epsilon}$ required by the global regularity analysis of Raugel and Sell in [23, 24, 25].

This paper is motivated by the desire to understand how the local anisotropic structure of the solution near the region of a potential singularity may lead to the depletion of vortex stretching, thus preventing the formation of a finite time singularity. To fully characterize the local solution structure of a potential singularity would be an extremely difficult task. Such study would shed useful light into our understanding of the dynamic depletion of vortex stretching and the global regularity of the Navier-Stokes equations. There has been some encouraging progress along this direction recently. In particular, Necas, Ruzicka and Sverak [20] and Tsai [28] have ruled out the possibility of an isotropic self-similar blow-up of the 3D Navier-Stokes equations. More recently, Chen-Strain-Tsai-Yau [5] prove that for axisymmetric 3D Navier-Stokes equations, if the velocity field $\mathbf{u}$ satisfies a scaling invariant blow-up rate

$$
|\mathbf{u}(x, t)| \leq C_{*} / \sqrt{r^{2}+(T-t)},
$$

where $r=\sqrt{x^{2}+y^{2}}$, then such blow-up is not possible at $t=T$. This to some extent excludes the locally self-similar isotropic blow-up of the axisymmetric Navier-Stokes equations. A related result with a stronger assumption can be found in [12] for the 3D Navier-Stokes equations.

There have been also some numerical evidences on the anisotropic scaling of the solution near the region of a potential blow-up of the 3D Euler and Navier-Stokes equations, see e.g. $[14,1,21,7,22,15,10,11]$. In particular, the recent work of Kerr [15] and Hou-Li [10, 11] gives some detailed description of the anisotropic scaling of the vorticity field near the inner core region of the maximum vorticity for two slightly perturbed antiparallel vortex tubes. The motivation for considering the rescaled initial data (2) and (3) is to understand whether such locally anisotropic blow-up is possible for the Navier-Stokes equations.

We remark that Hou-Li have recently proved the global regularity of the 3D axisymmetric Navier-Stokes equations with some large anisotropic initial data [9]. The results obtained in this paper complement those presented in [9]. For more discussions on the axisymmetric solutions of the Navier-Stokes equations, we refer to [4] and [5] and the references cited there. The 2D Boussinesq equations are closely related to the 3D axisymmetric NavierStokes equations with swirl (away from the symmetry axis). Recently, Hou-Li [8] and Chae [3] have proved independently the global existence of the $2 \mathrm{D}$ viscous Boussinesq equations with viscosity entering only in the fluid equation, but the density equation remains inviscid.

The remaining part of the paper is organized as follows. We give the formulation of the problem in Section 2. In Section 3, we prove a useful property of Riesz operators in $\mathbb{R}^{4} \times \mathbf{T}^{\mathbf{1}}$, where $\mathbf{T}^{\mathbf{1}}$ is the one-dimensional torus with periodicity 1 . This property is needed to prove our global regularity analysis of the axisymmetric Navier-Stokes equations with anisotropic 
initial data. Finally, we will present and prove the main result of this paper in Section 4 .

\section{Formulation}

Consider the 3D axi-symmetric incompressible Navier-Stokes equations with swirl.

$$
\left\{\begin{array}{l}
\mathbf{u}_{t}+(\mathbf{u} \cdot \nabla) \mathbf{u}=-\nabla p+\Delta \mathbf{u}, \\
\nabla \cdot \mathbf{u}=0, \\
\left.\mathbf{u}\right|_{t=0}=\mathbf{u}_{0}(\mathbf{x}), \quad \mathbf{x}=(x, y, z)
\end{array}\right.
$$

Let

$$
\mathbf{e}_{r}=\left(\frac{x}{r}, \frac{y}{r}, 0\right), \mathbf{e}_{\theta}=\left(-\frac{y}{r}, \frac{x}{r}, 0\right), \mathbf{e}_{z}=(0,0,1),
$$

be three unit vectors along the radial, the angular, and the $z$ directions respectively, $r=$ $\sqrt{x^{2}+y^{2}}$. We will decompose the velocity field as follows:

$$
\mathbf{u}=v^{r}(r, z, t) \mathbf{e}_{r}+u^{\theta}(r, z, t) \mathbf{e}_{\theta}+v^{z}(r, z, t) \mathbf{e}_{z} .
$$

In the above expression, $u^{\theta}$ is called the swirl component of the velocity field $\vec{u}$. The vorticity field can be expressed similarly

$$
\boldsymbol{\omega}=-\left(u^{\theta}\right)_{z}(r, z, t) \mathbf{e}_{r}+\omega^{\theta}(r, z, t) \mathbf{e}_{\theta}+\frac{1}{r}\left(r u^{\theta}\right)_{r}(r, z, t) \mathbf{e}_{z}
$$

where $\omega^{\theta}=v_{z}^{r}-v_{r}^{z}$.

One can derive evolution equations for $u^{\theta}$ and $\omega^{\theta}$ as follows (see e.g. [19, 4]).

$$
\begin{aligned}
& u_{t}^{\theta}+v^{r} u_{r}^{\theta}+v^{z} u_{z}^{\theta}=\left(\nabla^{2}-\frac{1}{r^{2}}\right) u^{\theta}-\frac{1}{r} v^{r} u^{\theta}, \\
& \omega_{t}^{\theta}+v^{r} \omega_{r}^{\theta}+v^{z} \omega_{z}^{\theta}=\left(\nabla^{2}-\frac{1}{r^{2}}\right) \omega^{\theta}+\frac{1}{r}\left(\left(u^{\theta}\right)^{2}\right)_{z}+\frac{1}{r} v^{r} \omega^{\theta}, \\
& -\left(\nabla^{2}-\frac{1}{r^{2}}\right) \psi^{\theta}=\omega^{\theta},
\end{aligned}
$$

where $\psi^{\theta}$ is the angular component of the stream function, $v^{r}$ and $v^{z}$ can be expressed in terms of the angular stream function $\psi^{\theta}$ as follows:

$$
v^{r}=-\frac{\partial \psi^{\theta}}{\partial z}, \quad v^{z}=\frac{1}{r} \frac{\partial}{\partial r}\left(r \psi^{\theta}\right)
$$

and $\nabla^{2}$ is defined as

$$
\nabla^{2}=\partial_{r}^{2}+\frac{1}{r} \partial_{r}+\partial_{z}^{2}
$$

Note that equations (7)-(9) completely determine the evolution of the 3D axisymmetric Navier-Stokes equations once the initial condition is given. 
By the well-known Caffarelli-Kohn-Nirenberg theory [2], the singularity set of any suitable weak solution of the 3D Navier-Stokes equations has one-dimensional Hausdorff measure zero. Thus, in the case of axisymmetric 3D Navier-Stokes equations with swirl, if there is any singularity, it must be along the symmetry axis, i.e. the $z$-axis. Therefore, we should focus our effort to understand the possible singular behavior of the 3D Navier-Stokes equations near the symmetry axis at $r=0$.

As observed by Liu and Wang in [18], any smooth solution of the 3D axisymmetric Navier-Stokes equations must satisfy the following compatibility condition at $r=0$ :

$$
u^{\theta}(0, z, t)=\omega^{\theta}(0, z, t)=\psi^{\theta}(0, z, t)=0 .
$$

Thus we can rewrite $u, \omega$ and $\psi$ as follows:

$$
u^{\theta}(r, z, t)=r u_{1}(r, z, t), \quad \omega^{\theta}(r, z, t)=r \omega_{1}(r, z, t), \quad \psi^{\theta}(r, z, t)=r \psi_{1}(r, z, t) .
$$

In [9], Hou-Li have derived the following equivalent system for $\left(u_{1}, \omega_{1}, \psi_{1}\right)$ :

$$
\begin{aligned}
& \left(u_{1}\right)_{t}+v^{r}\left(u_{1}\right)_{r}+v^{z}\left(u_{1}\right)_{z}=2 \psi_{1 z} u_{1}+\left(u_{1 z z}+u_{1 r r}+\frac{3 u_{1 r}}{r}\right) \\
& \left(\omega_{1}\right)_{t}+v^{r}\left(\omega_{1}\right)_{r}+v^{z}\left(\omega_{1}\right)_{z}=\left(\left(u_{1}\right)^{2}\right)_{z}+\left(\omega_{1 z z}+\omega_{1 r r}+\frac{3 \omega_{1 r}}{r}\right) \\
& -\left(\psi_{1 z z}+\psi_{1 r r}+\frac{3 \psi_{1 r}}{r}\right)=\omega_{1},
\end{aligned}
$$

where $v^{r}$ and $v^{z}$ are defined as

$$
v^{r}=-\frac{\partial\left(r \psi_{1}\right)}{\partial z}, \quad v^{z}=\frac{1}{r} \frac{\partial}{\partial r}\left(r^{2} \psi_{1}\right) .
$$

We note that the incompressibility condition implies that

$$
\left(r v^{r}\right)_{r}+\left(r v^{z}\right)_{z}=0
$$

which can be verified directly from (17).

\section{$3 \quad$ A Useful Property of Riesz operators on $\mathbb{R}^{4} \times \mathbf{T}^{\mathbf{1}}$}

Before we present our global regularity analysis, we would like to state and prove two technical lemmas regarding the property of a Riesz operator on $\mathbb{R}^{4} \times \mathbf{T}^{\mathbf{1}}$, where $\mathbb{T}^{1}$ is the one-dimensional torus with periodicity 1 . We first recall the following weighted CalderonZygmund inequality for a singular integral operator with a weight function which is in the $A_{p}$ class (see Stein [26] pp. $194-217$ for details). Let $K$ be a Riesz operator in $\mathbb{R}^{n}$ and $w(x)$ be a weight in the $A_{p}$ class (see page 194 of [26] for definition). One can extend the Calderon-Zygmund inequality for the singular integral operator with the integral having weight function $w(x)$. Specifically, for $1<p<\infty$, there exists a uniform constant $C$ such that

$$
\int_{\mathbb{R}^{n}}|K * f|^{p} w(x) d x \leq C^{p} \int_{\mathbb{R}^{n}}|f|^{p} w(x) d x,
$$


for all $f \in L^{p}\left(\mathbb{R}^{n}\right)$.

In this section, we prove a similar property for a Riesz operator on $\mathbb{R}^{4} \times \mathbf{T}^{\mathbf{1}}$. Let $\Delta=$ $\Sigma_{i=1}^{4} \partial_{x_{i}}^{2}+\partial_{z}^{2}$. Assume that $f(r, z)$ belongs to the weighted $L^{p}\left(\mathbb{R}^{4} \times \mathbb{T}^{1}\right)$ space with a weight function $w(x)=\frac{1}{r^{2}}$. Let $u=u(r, z), r=\sqrt{x_{1}^{2}+x_{2}^{2}+x_{3}^{2}+x_{4}^{2}}$, be the solution of

$$
-\Delta u=f
$$

with periodic boundary conditions along the $z$ direction with period 1 . We will prove the following lemma:

Lemma 1. Assume that $w(x)=w(r)$ belongs to the $A_{p}$ class in $\mathbb{R}^{5}$. Then there exists a uniform positive constant $C>0$ such that

$$
\int_{\mathbb{R}^{n-1} \times \mathbf{T}^{\mathbf{1}}}\left|\nabla^{2} u\right|^{p} w(x) d x_{1} \cdots d x_{n-1} d z \leq C \int_{\mathbb{R}^{n-1} \times \mathbf{T}^{\mathbf{1}}}|f|^{p} w(x) d x_{1} \cdots d x_{n-1} d z,
$$

provided that $f$ and $u$ are both $L^{p}$ integrable with the weight $w(x)$ for $1<p<\infty$.

Proof of Lemma 1. Let $\lambda>0$ and denote the one-dimensional torus with periodicity $\frac{1}{\lambda}$ by $\frac{1}{\lambda} \mathbb{T}^{1}$. For $\left(x_{1}, \cdots, x_{4}, z\right) \in \mathbb{R}^{4} \times \frac{1}{\lambda} \mathbb{T}^{1}$, define

$$
\left\{\begin{array}{l}
u^{\lambda}\left(x_{1}, \cdots, x_{4}, z\right)=u\left(\lambda x_{1}, \cdots, \lambda x_{4}, \lambda z\right), \\
f^{\lambda}\left(x_{1}, \cdots, x_{4}, z\right)=\lambda^{2} f\left(\lambda x_{1}, \cdots, \lambda x_{4}, \lambda z\right) .
\end{array}\right.
$$

It is easy to see that

$$
-\Delta u^{\lambda}=f^{\lambda}, \quad\left(x_{1}, \cdots, x_{4}, z\right) \in \mathbb{R}^{4} \times \frac{1}{\lambda} \mathbb{T}^{1} .
$$

We claim that there exists a uniform constants $C_{1}$ and $C_{2}$ independent of $\lambda$ such that

$$
\left\|\left(\nabla^{2} u^{\lambda}\right) w(x)^{\frac{1}{p}}\right\|_{L^{p}\left(\mathbb{R}^{4} \times \frac{1}{\lambda} \mathbb{T}^{1}\right)} \leq C_{1}\left\|f^{\lambda} w(x)^{\frac{1}{p}}\right\|_{L^{p}\left(\mathbb{R}^{4} \times \frac{1}{\lambda} \mathbb{T}^{1}\right)}+C_{2}\left\|u^{\lambda} w(x)^{\frac{1}{p}}\right\|_{L^{p}\left(\mathbb{R}^{4} \times \frac{1}{\lambda} \mathbb{T}^{1}\right)} .
$$

To prove this, we introduce a smooth cutoff function $\phi(z)$ which satisfies $0 \leq \phi(z) \leq 1$, $\phi(z)=0$ for $z \leq-1$ or $z \geq 2$, and $\phi(z)=1$ for $0 \leq z \leq 1$. For $\lambda \in(0,1]$, we define $\phi^{\lambda}(z)=\phi(\lambda z)$. A simple computation gives

$$
-\Delta\left(\phi^{\lambda} u^{\lambda}\right)=\phi^{\lambda} f^{\lambda}-2 \nabla \phi^{\lambda} \cdot \nabla u^{\lambda}-u^{\lambda} \Delta \phi^{\lambda}, \quad\left(x_{1}, \cdots, x_{4}, z\right) \in \mathbb{R}^{5} .
$$

Since $w(x)$ belongs to the $A_{p}$ class, the weighted Calderon-Zygmund inequality in $\mathbb{R}^{n}$ (see (19)) implies that

$$
\begin{aligned}
\left\|\left(\nabla^{2} u^{\lambda}\right) w(x)^{\frac{1}{p}}\right\|_{L^{p}\left(\mathbb{R}^{4} \times \frac{1}{\lambda} \mathbb{T}^{1}\right)} \leq\left\|\nabla^{2}\left(\phi^{\lambda} u^{\lambda}\right) w(x)^{\frac{1}{p}}\right\|_{L^{p}\left(\mathbb{R}^{5}\right)} \\
\leq C\left\|\left(\phi^{\lambda} f^{\lambda}-2 \nabla \phi^{\lambda} \cdot \nabla u^{\lambda}-u^{\lambda} \Delta \phi^{\lambda}\right) w(x)^{\frac{1}{p}}\right\|_{L^{p}\left(\mathbb{R}^{5}\right)} \\
\leq C\left(\left\|f^{\lambda} w(x)^{\frac{1}{p}}\right\|_{L^{p}\left(\mathbb{R}^{4} \times \frac{1}{\lambda} \mathbb{T}^{1}\right)}+\lambda^{2}\left\|u^{\lambda} w(x)^{\frac{1}{p}}\right\|_{L^{p}\left(\mathbb{R}^{4} \times \frac{1}{\lambda} \mathbb{T}^{1}\right)}\right) \\
\quad+\frac{1}{2}\left\|\left(\nabla^{2} u^{\lambda}\right) w(x)^{\frac{1}{p}}\right\|_{L^{p}\left(\mathbb{R}^{4} \times \frac{1}{\lambda} \mathbb{T}^{1}\right)},
\end{aligned}
$$


where we have used the following estimate (note that $\phi=\phi(z), w(x)=w(r)$ and $u(x)=$ $u(r, z))$ :

$$
\begin{aligned}
& \left\|\nabla \phi^{\lambda} \cdot \nabla u^{\lambda} w(x)^{\frac{1}{p}}\right\|_{L^{p}\left(\mathbb{R}^{5}\right)} \leq C \lambda\left(\int_{\mathbb{R}^{4} \times \frac{1}{\lambda} \mathbb{T}^{1}}\left|\partial_{z} u^{\lambda}\right|^{p} w(x) d x\right)^{\frac{1}{p}} \\
& \leq C \lambda\left(\int_{\mathbb{R}} w(r) r^{3} d r \int_{\frac{1}{\lambda} \mathbb{T}^{1}}\left|\partial_{z} u^{\lambda}\right|^{p} d z\right)^{\frac{1}{p}} \\
& \leq C_{p} \lambda^{2}\left\|u^{\lambda} w(x)^{\frac{1}{p}}\right\|_{L^{p}\left(\mathbb{R}^{4} \times \frac{1}{\lambda} \mathbb{T}^{1}\right)}+\frac{1}{4}\left\|\left(\partial_{z}^{2} u^{\lambda}\right) w(x)^{\frac{1}{p}}\right\|_{L^{p}\left(\mathbb{R}^{4} \times \frac{1}{\lambda} \mathbb{T}^{1}\right)} .
\end{aligned}
$$

In the last step of the above estimate, we have used the one-dimensional Sobolev inequality along the $z$-direction:

$$
\left\|u_{z}\right\|_{L^{p}\left(\frac{1}{\lambda} \mathbb{T}^{1}\right)}^{p} \leq \frac{C_{p} \lambda}{C}\|u\|_{L^{p}\left(\frac{1}{\lambda} \mathbb{T}^{1}\right)}^{p}+\frac{1}{4 C \lambda}\left\|\partial_{z}^{2} u\right\|_{L^{p}\left(\frac{1}{\lambda} \mathbb{T}^{1}\right)}^{p},
$$

for some positive constant $C_{p}$ depending on $p$. Thus, one has

$$
\left\|\left(\nabla^{2} u^{\lambda}\right) w(x)^{\frac{1}{p}}\right\|_{L^{p}\left(\mathbb{R}^{4} \times \frac{1}{\lambda} \mathbb{T}^{1}\right)} \leq C\left(\left\|f^{\lambda} w(x)^{\frac{1}{p}}\right\|_{L^{p}\left(\mathbb{R}^{5}\right)}+\lambda^{2}\left\|u^{\lambda} w(x)^{\frac{1}{p}}\right\|_{L^{p}\left(\mathbb{R}^{4} \times \frac{1}{\lambda} \mathbb{T}^{1}\right)}\right)
$$

for some uniform positive constant $C>0$. For $\lambda>1$, we denote $N$ by the biggest integer less that $\lambda$ and compute

$$
-\Delta\left(\phi u^{\lambda}\right)=\phi f^{\lambda}-2 \nabla \phi \cdot \nabla u^{\lambda}-u^{\lambda} \Delta \phi, \quad\left(x_{1}, \cdots, x_{4}, z\right) \in \mathbb{R}^{5} .
$$

Similarly, by using the weighted Calderon-Zygmund inequality in $\mathbb{R}^{5}$, we obtain

$$
\begin{aligned}
& N\left\|\left(\nabla^{2} u^{\lambda}\right) w(x)^{\frac{1}{p}}\right\|_{L^{p}\left(\mathbb{R}^{4} \times \frac{1}{\lambda} \mathbb{T}^{1}\right)} \leq C\left\|\nabla^{2}\left(\phi u^{\lambda}\right) w(x)^{\frac{1}{p}}\right\|_{L^{p}\left(\mathbb{R}^{5}\right)} \\
& \leq C\left\|\left(\phi f^{\lambda}-2 \nabla \phi \cdot \nabla u^{\lambda}-u^{\lambda} \Delta \phi\right) w(x)^{\frac{1}{p}}\right\|_{L^{p}\left(\mathbb{R}^{5}\right)} \\
& \leq C N\left(\left\|f^{\lambda} w(x)^{\frac{1}{p}}\right\|_{L^{p}\left(\mathbb{R}^{4} \times \frac{1}{\lambda} \mathbb{T}^{1}\right)}+\left\|u^{\lambda} w(x)^{\frac{1}{p}}\right\|_{L^{p}\left(\mathbb{R}^{4} \times \frac{1}{\lambda} \mathbb{T}^{1}\right)}\right) \\
& \quad+\frac{N}{2}\left\|\left(\nabla^{2} u^{\lambda}\right) w(x)^{\frac{1}{p}}\right\|_{L^{p}\left(\mathbb{R}^{4} \times \frac{1}{\lambda} \mathbb{T}^{1}\right)},
\end{aligned}
$$

which gives

$$
\left\|\left(\nabla^{2} u^{\lambda}\right) w(x)^{\frac{1}{p}}\right\|_{L^{p}\left(\mathbb{R}^{4} \times \frac{1}{\lambda} \mathbb{T}^{1}\right)} \leq C\left(\left\|f^{\lambda} w(x)^{\frac{1}{p}}\right\|_{L^{p}\left(\mathbb{R}^{4} \times \frac{1}{\lambda} \mathbb{T}^{1}\right)}+\left\|u^{\lambda} w(x)^{\frac{1}{p}}\right\|_{L^{p}\left(\mathbb{R}^{4} \times \frac{1}{\lambda} \mathbb{T}^{1}\right)}\right) .
$$

By (25) and (26), we prove the claim (23).

Note that

$$
\left\{\begin{array}{l}
\left\|\left(\nabla^{2} u^{\lambda}\right) w(x)^{\frac{1}{p}}\right\|_{L^{p}\left(\mathbb{R}^{4} \times \frac{1}{\lambda} \mathbb{T}^{1}\right)}=\lambda^{2-\frac{5}{p}}\left\|\left(\nabla^{2} u\right) w(x)^{\frac{1}{p}}\right\|_{L^{p}\left(\mathbb{R}^{4} \times \mathbb{T}^{1}\right)}, \\
\left\|f^{\lambda} w(x)^{\frac{1}{p}}\right\|_{L^{p}\left(\mathbb{R}^{4} \times \frac{1}{\lambda} \mathbb{T}^{1}\right)}=\lambda^{2-\frac{5}{p}}\left\|f w(x)^{\frac{1}{p}}\right\|_{L^{p}\left(\mathbb{R}^{4} \times \mathbb{T}^{1}\right)}, \\
\left\|u^{\lambda} w(x)^{\frac{1}{p}}\right\|_{L^{p}\left(\mathbb{R}^{4} \times \frac{1}{\lambda} \mathbb{T}^{1}\right)}=\lambda^{-\frac{5}{p}}\left\|u w(x)^{\frac{1}{p}}\right\|_{L^{p}\left(\mathbb{R}^{4} \times \mathbb{T}^{1}\right)} .
\end{array}\right.
$$


Thus, we deduce from (23) that

$$
\left\|\left(\nabla^{2} u\right) w(x)^{\frac{1}{p}}\right\|_{L^{p}\left(\mathbb{R}^{4} \times \mathbb{T}^{1}\right)} \leq C_{1}\left\|f w(x)^{\frac{1}{p}}\right\|_{L^{p}\left(\mathbb{R}^{4} \times \mathbb{T}^{1}\right)}+\frac{C_{2}}{\lambda^{2}}\left\|u w(x)^{\frac{1}{p}}\right\|_{L^{p}\left(\mathbb{R}^{4} \times \mathbb{T}^{1}\right)} .
$$

By letting $\lambda \rightarrow \infty$ in (27), we prove the lemma.

Now we prove the following Lemma, which plays an important role in our global regularity analysis:

Lemma 2. Assume that $\omega_{1}$ and $\psi_{1}$ are in $L^{p}\left(\mathbb{R}^{2} \times \mathbb{T}^{1}\right)$ with $1<p<\infty$, and $\psi_{1}$ is the solution of (16) with periodic boundary condition along the z-direction with period 1 . Then we have

$$
\left\|\psi_{1 z z}\right\|_{L^{p}\left(\mathbb{R}^{2} \times \mathbb{T}^{1}\right)} \leq C\left\|\omega_{1}\right\|_{L^{p}\left(\mathbb{R}^{2} \times \mathbb{T}^{1}\right)}
$$

for $1<p<\infty$.

Proof of Lemma 2. To prove (28), we observe that

$$
\Delta=\frac{\partial^{2}}{\partial z^{2}}+\frac{\partial^{2}}{\partial r^{2}}+3 \frac{\partial}{\partial r}
$$

is an axisymmetric Laplacian operator in five space dimensions, where $r=\sqrt{x_{1}^{2}+x_{2}^{2}+x_{3}^{3}+x_{4}^{2}}$ for $x=\left(x_{1}, x_{2}, x_{3}, x_{4}, z\right) \in R^{5}$. Let $n=5$. Since $-\Delta \psi_{1}=\omega_{1}$, Lemma 1 implies that

$$
\int_{\mathbb{R}^{4} \times \mathbf{T}^{\mathbf{1}}}\left|\partial_{z}^{2} \psi_{1}\right|^{p} w(x) d x_{1} \cdots d x_{4} d z \leq C \int_{\mathbb{R}^{4} \times \mathbf{T}^{\mathbf{1}}}\left|\omega_{1}\right|^{p} w(x) d x_{1} \cdots d x_{4} d z .
$$

provided that $w(x)=\frac{1}{r^{2}}$ belongs to the $A_{p}$ class. We will show that $w$ belongs to the $A_{p}$ class. In terms of the cylindrical coordinate, the above inequality (29) can be written as

$$
\left(\int_{0}^{1} \int_{0}^{\infty}\left|\psi_{1 z z}\right|^{p} r^{3} r^{-2} d r d z\right)^{\frac{1}{p}} \leq C\left(\int_{0}^{1} \int_{0}^{\infty}\left|\omega_{1}\right|^{p} r^{3} r^{-2} d r d z\right)^{\frac{1}{p}}
$$

which is exactly (28).

It remains to show that $w(x)=\frac{1}{r^{2}}$ belongs to the $A_{p}$ class. In the following, we will prove that $w(x)=\frac{1}{r^{\alpha}}$ belongs to the $A_{p}$ class for $0<\alpha<4$ (see page 194 of [26] for definition). To see this, let $B_{R}$ be a ball centered at $x=0$ with radius $R$. Denote by $\left|B_{R}\right|$ the volume of $B_{R}$, and $p^{\prime}$ be the conjugate of $p$, i.e. $\frac{1}{p}+\frac{1}{p^{\prime}}=1$. We have

$$
\begin{aligned}
& \left(\frac{1}{\left|B_{R}\right|} \int_{B_{R}} w(x) d x\right)\left(\frac{1}{\left|B_{R}\right|} \int_{B_{R}} w(x)^{-\frac{p^{\prime}}{p}} d x\right)^{\frac{p}{p^{\prime}}} \\
& \leq \frac{C}{R^{5}}\left(\int_{0}^{R} \int_{0}^{\sqrt{R^{2}-z^{2}}} r^{3-\alpha} d r d z\right)\left(\frac{1}{R^{5}} \int_{0}^{R} d z \int_{0}^{\sqrt{R^{2}-z^{2}}} r^{3+\alpha \frac{p^{\prime}}{p}} d r d z\right)^{\frac{p}{p^{\prime}}} \\
& \leq \frac{C}{R^{5}}\left(\int_{0}^{R}\left(R^{2}-z^{2}\right)^{\frac{4-\alpha}{2}} d z\right)\left(\frac{1}{R^{5}} \int_{0}^{R}\left(R^{2}-z^{2}\right)^{2+\frac{\alpha p^{\prime}}{2 p}} d z\right)^{\frac{p}{p^{\prime}}} \\
& \leq \frac{C}{R^{5}} R^{5-\alpha}\left(\frac{1}{R^{5}} R^{5+\alpha \frac{p^{\prime}}{p}}\right)^{\frac{p}{p^{\prime}}} \leq C R^{\alpha-\alpha}=C,
\end{aligned}
$$


independent of $R$ for $0<\alpha<4$. Similarly we can prove the same estimate for $B_{R}$ centered at any point. This completes the proof of Lemma 2.

\section{The main result and its proof}

Now we state our main result in this paper.

Theorem 1. Let $0<\delta<1$ be a given number which could be arbitrarily small. Assume that

$$
u^{\theta}(r, z, 0)=\frac{1}{\epsilon^{1-\delta}} U_{0}(\epsilon r, z), \quad \omega^{\theta}(r, z, 0)=\frac{1}{\epsilon^{1-\delta}} W_{0}(\epsilon r, z),
$$

where $U_{0}(r, \tilde{z})=r U_{1}(r, \tilde{z}), W_{0}(r, \tilde{z})=r W_{1}(r, \tilde{z})$, and $U_{0}, U_{1} \in L^{2 p}\left(R^{2} \times[0,1]\right), W_{0}, W_{1} \in$ $L^{2 q}\left(R^{2} \times[0,1]\right)$ with $p=2 q$ and $q>1 / \delta$. Further, we assume that the initial velocity field $\mathbf{u}_{0} \in L^{2}\left(R^{2} \times[0,1]\right)$ and the initial conditions for $\psi^{\theta}, u^{\theta}$ and $\omega^{\theta}$ are odd and periodic in $z$ with period 1 . Then there exists $\epsilon_{0}\left(\delta, U_{0}, W_{0}\right)>0$ such that for all $0<\epsilon \leq \epsilon_{0}$ the $3 D$ axisymmetric Navier-Stokes equations with initial data given by (31) have a unique global regular solution.

Remark 1. By using Theorem 1, we can easily obtain the global regularity of the initial data (2) by using a scaling argument. Specifically, if we denote by $u$ the solution of the Navier-Stokes equations with initial data $(31)$, then $u_{\epsilon}(\mathbf{x}, t) \equiv \frac{1}{\epsilon} u\left(\frac{\mathbf{x}}{\epsilon}, \frac{t}{\epsilon^{2}}\right)$ is the solution of the Navier-Stokes equations with the rescaled initial data (2). Thus the global regularity of the Navier-Stokes equations with initial data (2) follows immediately from Theorem 1. Using a slightly different rescaling, we can prove the global regularity of the Navier-Stokes equations with initial data (3). See Remark 4 at the end of this section for more detail.

Remark 2. It is interesting to derive the corresponding global bound for the initial data (2). Let $\left(u^{\theta}, \omega^{\theta}\right)$ be the solution corresponding to the initial data $(31)$, and $\left(u_{\epsilon}^{\theta}, \omega_{\epsilon}^{\theta}\right)$ be the solution corresponding to the initial data (2). If we denote by $u_{1}^{\epsilon}=u_{\epsilon}^{\theta} / r, \omega_{1}^{\epsilon}=\omega_{\epsilon}^{\theta} / r$, and $u_{1}=u^{\theta} / r, \omega_{1}=\omega^{\theta} / r$, then we have

$$
u_{1}^{\epsilon}(r, z, t)=\frac{1}{\epsilon^{2}} u_{1}\left(\frac{r}{\epsilon}, \frac{z}{\epsilon}, \frac{t}{\epsilon^{2}}\right), \quad \omega_{1}^{\epsilon}(r, z, t)=\frac{1}{\epsilon^{3}} \omega_{1}\left(\frac{r}{\epsilon}, \frac{z}{\epsilon}, \frac{t}{\epsilon^{2}}\right) .
$$

Using the above scaling relationship and substituting $f=u_{1}^{p}$ and $g=\omega_{1}^{q}$ into the global estimate (47), we obtain the following global estimate for $u_{1}^{\epsilon}$ and $\omega_{1}^{\epsilon}$ with the initial data (2):

$$
\begin{aligned}
\left\|u_{1}^{\epsilon}(t)\right\|_{L^{2 p}\left(\Omega_{\epsilon}\right)} \leq \epsilon^{-2+\delta+1 / 2 p}\left\|U_{1}\right\|_{L^{2 p}\left(\Omega_{\epsilon}\right)}+\left(2 / C_{q}\right)^{\frac{1}{2 p}} \epsilon^{-2+\delta / 2+1 / 2 p}\left\|W_{1}\right\|_{L^{2 q}\left(\Omega_{\epsilon}\right)}^{\frac{1}{2}} \\
\left\|\omega_{1}^{\epsilon}(t)\right\|_{L^{2 q}\left(\Omega_{\epsilon}\right)} \leq \epsilon^{-3+\delta+1 / 2 q}\left\|W_{1}\right\|_{L^{2 q}\left(\Omega_{\epsilon}\right)}+\left(C_{q} / 2\right)^{\frac{1}{2 q}} \epsilon^{-3+2 \delta+1 / 2 q}\left\|U_{1}\right\|_{L^{2 p}\left(\Omega_{\epsilon}\right)}^{2}
\end{aligned}
$$

where $\Omega_{\epsilon}=R^{2} \times[0, \epsilon]$. Note that the first term on the right hand side of (32) is equal to $\left\|u_{1}^{\epsilon}(0)\right\|_{L^{2 p}\left(\Omega_{\epsilon}\right)}$ and is smaller than the second term by a factor of $O\left(\epsilon^{\delta / 2}\right)$. This implies that $\left\|u_{\epsilon}^{\theta}\right\|_{L^{2 p}}$ may experience a rapid growth dynamically for $\epsilon$ small due to the contribution from the initial condition of $\omega_{\epsilon}^{\theta}$. Similarly, the first term on the right hand side of (33) is equal 
to $\left\|\omega_{1}^{\epsilon}(0)\right\|_{L^{2 p}\left(\Omega_{\epsilon}\right)}$, but is larger than the second term by a factor of $O\left(\epsilon^{-\delta}\right)$. Thus the growth of $\left\|\omega_{\epsilon}^{\theta}\right\|_{L^{2 q}}$ is more moderate. This phenomenon is similar to the solution behavior of the 1D model derived by Hou-Li in [9] where they show that $\left(u_{1 z}\right)^{2}+\omega_{1}^{2}$ satisfies a maximum principle. However, $u_{1}$ can experience large growth dynamically due to the contribution from $u_{1 z}$ and $\omega_{1}$ at $t=0$.

Remark 3. It is worthwhile to point out that all the functions involved are in fact smooth for $t>0$ based on our assumptions and the dynamic control that we obtain. Here, the key is the dynamic control of $\left\|u_{1}(t)\right\|_{L^{2 p}} \leq C$ and $\left\|w_{1}(t)\right\|_{L^{p}} \leq C$. With these two estimates, the solution can be smoothly extended to all times. More specifically, the work of [2] and [17] implies that the velocity field $\mathbf{u}(x, t)$ is square integrable and smooth in the region where $r>1$. Thus $\mathbf{u}(x, t)$ has a bounded $L^{p}$ norm in the region $r>1$ for $2 \leq p \leq \infty$. On the other hand, we have $\left|u^{\theta}\right| \leq C\left|u_{1}\right|$ and $\left|w^{\theta}\right| \leq C\left|w_{1}\right|$ in the region where $r$ is bounded. Therefore, our dynamic control of $\left\|u_{1}(t)\right\|_{L^{2 p}} \leq C$ (note that $2 p \geq 3$ by the assumption of Theorem 1) and $\left\|w_{1}(t)\right\|_{L^{p}} \leq C$ lead to the following a priori bound on the gradient of $u^{r} \mathbf{e}_{r}+u^{z} \mathbf{e}_{z}$ (see $[20])$ :

$$
\begin{aligned}
& \left\|\nabla\left(u^{r} \mathbf{e}_{r}+u^{z} \mathbf{e}_{z}\right)\right\|_{L^{p}\left(\mathbb{R}^{2} \times \mathbb{T}^{1}, r<1.5\right)} \\
& \leq C\left(\left\|\nabla \times\left(u^{r} \mathbf{e}_{r}+u^{z} \mathbf{e}_{z}\right)\right\|_{L^{p}\left(\mathbb{R}^{2} \times \mathbb{T}^{1}, r<2\right)}+\left\|\nabla \cdot\left(u^{r} \mathbf{e}_{r}+u^{z} \mathbf{e}_{z}\right)\right\|_{L^{p}\left(\mathbb{R}^{2} \times \mathbb{T}^{1}, r<2\right)}\right. \\
& \left.\quad+\left\|u^{r} \mathbf{e}_{r}+u^{z} \mathbf{e}_{z}\right\|_{L^{2}\left(\mathbb{R}^{2} \times \mathbb{T}^{1}, r<2\right)}\right) \\
& \leq C\left(\left\|\omega^{\theta} \mathbf{e}_{\theta}\right\|_{L^{p}\left(\mathbb{R}^{2} \times \mathbb{T}^{1}, r<2\right)}+\left\|u^{r} \mathbf{e}_{r}+u^{z} \mathbf{e}_{z}\right\|_{L^{2}\left(\mathbb{R}^{2} \times \mathbb{T}^{1}, r<2\right)}\right) \\
& <\infty
\end{aligned}
$$

where we have used the fact that $\omega^{\theta} \mathbf{e}_{\theta}=\nabla \times\left(u^{r} \mathbf{e}_{r}+u^{z} \mathbf{e}_{z}\right)$ and $\nabla \cdot\left(u^{r} \mathbf{e}_{r}+u^{z} \mathbf{e}_{z}\right)=0$. The Sobolev embedding theory and the fact that $u^{r} \mathbf{e}_{r}+u^{z} \mathbf{e}_{z} \in L^{2}\left(\mathbb{R}^{2} \times \mathbb{T}^{1}, r<2\right)$ imply that $u^{r} \mathbf{e}_{r}+u^{z} \mathbf{e}_{z} \in L^{3}\left(\mathbb{R}^{2} \times \mathbb{T}^{1}, r<1.5\right)$ since $p \geq \frac{3}{2}$. Then the well-known regularity result (see [13]) implies that $\mathbf{u}(t)$ is smooth and $L^{p}$ integrable with $p \geq 2$ for $t>0$.

Proof of Theorem 1. By the assumptions of Theorem 1, we know that the initial velocity field $\mathbf{u}_{0} \in L^{2}\left(R^{2} \times[0,1]\right)$ and that $\left.u^{\theta}\right|_{t=0} \in L^{2 p}\left(R^{2} \times[0,1]\right),\left.\omega^{\theta}\right|_{t=0} \in L^{2 q}\left(R^{2} \times[0,1]\right)$ with $p=2 q$ and $q>1 / \delta$. Using these properties of the initial data, one can show that there exists a finite time $T>0$ such that the axisymmetric Navier-Stokes has a unique regular solution for $0<t \leq T$. In the rest of the proof, we will perform a dynamic estimate for the regular solution for $0<t \leq T$ and show that $T$ can be made arbitrarily large.

Denote $\Omega=R^{2} \times[0,1]$. Define $f=\left|u_{1}\right|^{p}$ and $g=\left|\omega_{1}\right|^{q}$, with $p=2 q$. Multiplying (14) by $\left|u_{1}\right|^{2 p-2} u_{1}$ and integrating over $\Omega$, we get after using (18) that

$$
\frac{1}{2 p} \frac{d}{d t} \int_{\Omega} f^{2} r d r d z=\int_{\Omega} \psi_{1 z}\left|u_{1}\right|^{2 p} r d r d z+\int_{\Omega}\left|u_{1}\right|^{2 p-2} u_{1}\left(u_{1 z z}+u_{1 r r}+\frac{3 u_{1 r}}{r}\right) r d r d z .
$$


Note that

$$
\begin{aligned}
\int_{\Omega}\left|u_{1}\right|^{2 p-2} u_{1} u_{1 z z} r d r d z & =-(2 p-1) \int_{\Omega}\left|u_{1}\right|^{2 p-2}\left(\left|u_{1}\right|_{z}\right)^{2} r d r d z \\
& =-(2 p-1) \int_{\Omega}\left(\left|u_{1}\right|^{p-1}\left|u_{1}\right|_{z}\right)^{2} r d r d z \\
& =-\frac{(2 p-1)}{p^{2}} \int_{\Omega}\left(f_{z}\right)^{2} r d r d z .
\end{aligned}
$$

Similarly, we obtain

$$
\begin{aligned}
\int_{\Omega}\left|u_{1}\right|^{2 p-2} u_{1} \frac{\left(r u_{1 r}\right)_{r}}{r} r d r d z & =\int_{\Omega}\left|u_{1}\right|^{2 p-2} u_{1}\left(r u_{1 r}\right)_{r} d r d z \\
& =-(2 p-1) \int_{\Omega}\left|u_{1}\right|^{2 p-2}\left(\left|u_{1}\right|_{r}\right)^{2} r d r d z \\
& =-\frac{(2 p-1)}{p^{2}} \int_{\Omega}\left(f_{r}\right)^{2} r d r d z
\end{aligned}
$$

and

$$
\begin{aligned}
\int_{\Omega}\left|u_{1}\right|^{2 p-2} u_{1} \frac{2 u_{1 r}}{r} r d r d z & =2 \int_{\Omega}\left|u_{1}\right|^{2 p-2} u_{1} u_{1 r} d r d z=\frac{1}{p} \int_{\Omega}\left(\left|u_{1}\right|^{2 p}\right)_{r} d r d z \\
& =-\frac{1}{p} \int_{0}^{1}\left|u_{1}\right|^{2 p}(0, z, t) d z=-\frac{1}{p} \int_{0}^{1} f^{2}(0, z, t)^{2} d z
\end{aligned}
$$

Therefore, we have

$$
\int_{\Omega}\left|u_{1}\right|^{2 p-2} u_{1}\left(u_{1 z z}+u_{1 r r}+\frac{3 u_{1 r}}{r}\right) r d r d z=-\frac{(2 p-1)}{p^{2}} \int_{\Omega}|\nabla f|^{2} r d r d z-\frac{1}{p} \int_{0}^{1} f^{2}(0, z, t)^{2} d z .(35
$$

On the other hand, if we let $\tilde{p}=2 q, \tilde{q}=\tilde{p} /(\tilde{p}-1)$, we get

$$
\begin{aligned}
\int_{\Omega} \psi_{1 z} f^{2} r d r d z & \leq\left\|\psi_{1 z}\right\|_{L^{\tilde{p}}(\Omega)}\left\|f^{2}\right\|_{L^{\tilde{q}}(\Omega)} \leq\left\|\psi_{1 z z}\right\|_{L^{\tilde{p}}(\Omega)}\left\|f^{2}\right\|_{L^{\tilde{q}}(\Omega)} \\
& \leq C\left\|\omega_{1}\right\|_{L^{\tilde{p}}(\Omega)}\left\|f^{2}\right\|_{L^{\tilde{q}}(\Omega)}=C\|g\|_{L^{2}(\Omega)}^{\frac{1}{q}}\|f\|_{L^{\frac{4 q}{2 q-1}(\Omega)}}^{2}
\end{aligned}
$$

where we have used $\left\|\psi_{1 z}\right\|_{L^{\tilde{p}}(\Omega)} \leq\left\|\psi_{1 z z}\right\|_{L^{\tilde{p}}(\Omega)}$ which follows from the Poincare inequality along the $z$-direction, and the weighted Calderon-Zygmund estimate $\left\|\psi_{1 z z}\right\|_{L^{\tilde{p}}(\Omega)} \leq C\left\|\omega_{1}\right\|_{L \tilde{p}(\Omega)}$, which we have proved in Lemma 2. In order to apply Lemma 2, we need to show that $\psi_{1} \in L^{\tilde{p}}(\Omega)$. To see this, we use the fact that $V^{r}=-\frac{\partial}{\partial z} \psi^{\theta} \in L^{m}$ for any $m \geq 2$ and $\psi^{\theta}$ is odd. Thus we can apply the Poincare inequality along $z$-direction to show that $\psi^{\theta} \in L^{m}(\Omega)$, which implies that $\psi_{1}=\frac{1}{r} \psi^{\theta} \in L^{s}(\Omega)$ for any $s>1$ in the region $r>1$. In the region where $r$ is bounded and $0<z<2$, the interior estimate of the general elliptic theory implies that $\psi_{1} \in L^{\tilde{p}}(\Omega)$ whenever $\omega_{1} \in L^{\tilde{p}}(\Omega)$. 
Using the Sobolev interpolation inequality,

$$
\|f\|_{L^{\frac{4 q}{2 q-1}}} \leq C\|f\|_{L^{2}}^{\alpha}\|\nabla f\|_{L^{2}}^{1-\alpha}
$$

with $\alpha=1-\frac{3}{4 q}$ and $q \geq 1$, we obtain

$$
\frac{1}{2 p} \frac{d}{d t} \int f^{2} r d r d z \leq C_{p}\|g\|_{L^{2}}^{\frac{1}{q}}\|f\|_{L^{2}}^{2-\frac{3}{2 q}}\|\nabla f\|_{L^{2}}^{\frac{3}{2 q}}-\frac{(2 p-1)}{p^{2}}\|\nabla f\|_{L^{2}}^{2} .
$$

By the assumption, the initial conditions for $\psi_{1}, u_{1}$, and $\omega_{1}$ are odd functions in $z$. This oddness property of the solution is preserved dynamically. Thus we have $f(r, 0, t)=$ $g(r, 0, t)=0$. By using the Poincare inequality along the $z$-direction, we obtain

$$
\|f\|_{L^{2}(\Omega)} \leq\left\|f_{z}\right\|_{L^{2}(\Omega)}, \quad\|g\|_{L^{2}(\Omega)} \leq\left\|g_{z}\right\|_{L^{2}(\Omega)} .
$$

It follows from (38) and (37) that

$$
\frac{1}{2 p} \frac{d}{d t} \int_{\Omega} f^{2} r d r d z \leq C_{p}\|g\|_{L^{2}(\Omega)}^{\frac{1}{q}}\|\nabla f\|_{L^{2}(\Omega)}^{2}-\frac{(2 p-1)}{p^{2}}\|\nabla f\|_{L^{2}(\Omega)}^{2} .
$$

Next, we multiply $\left|\omega_{1}\right|^{2 q-2} \omega_{1}$ to (15) and integrate over $\Omega$. We have

$$
\begin{aligned}
\frac{1}{2 q} \frac{d}{d t} \int_{\Omega} g^{2} r d r d z & \leq \int_{\Omega}\left(u_{1}^{2}\right)_{z}\left|\omega_{1}\right|^{2 q-2} \omega_{1} r d r d z-\frac{(2 q-1)}{q^{2}}\|\nabla g\|_{L^{2}(\Omega)}^{2} \\
& \leq \int_{\Omega}\left(f^{\frac{2}{p}}\right)_{z}\left|\omega_{1}\right|^{2 q-2} \omega_{1} r d r d z-\frac{(2 q-1)}{q^{2}}\|\nabla g\|_{L^{2}(\Omega)}^{2} \\
& \leq\left(2-\frac{1}{q}\right) \int_{\Omega} f^{\frac{2}{p}} g^{1-\frac{1}{q}}\left|g_{z}\right| r d r d z-\frac{(2 q-1)}{q^{2}}\|\nabla g\|_{L^{2}(\Omega)}^{2} \\
& \leq\left(2-\frac{1}{q}\right)\left\|g_{z}\right\|_{L^{2}(\Omega)}\left(\int_{\Omega} f^{\frac{4}{p}} g^{2\left(1-\frac{1}{q}\right)} r d r d z\right)^{1 / 2}-\frac{(2 q-1)}{q^{2}}\|\nabla g\|_{L^{2}(\Omega)}^{2} .
\end{aligned}
$$

Let $\tilde{p}=p / 2, \tilde{q}=\frac{\tilde{p}}{\tilde{p}-1}=\frac{p}{p-2}$. Then we obtain by using the Hölder inequality that

$$
\begin{aligned}
\left(\int_{\Omega} f^{\frac{4}{p}} g^{2\left(1-\frac{1}{q}\right)} r d r d z\right)^{1 / 2} & \leq\left(\int_{\Omega} f^{2} r d r d z\right)^{1 / p}\left(\int_{\Omega} g^{2\left(1-\frac{1}{q}\right) \frac{p}{p-2}} r d r d z\right)^{\frac{p-2}{2 p}} \\
& \leq\|f\|_{L^{2}(\Omega)}^{\frac{2}{p}}\|g\|_{L^{2}(\Omega)}^{1-\frac{2}{p}}
\end{aligned}
$$

where we have used the relationship $p=2 q$. Therefore, we obtain

$$
\begin{aligned}
\frac{1}{2 q} \frac{d}{d t} \int_{\Omega} g^{2} r d r d z & \leq\left(2-\frac{1}{q}\right)\left\|g_{z}\right\|_{L^{2}(\Omega)}\|f\|_{L^{2}(\Omega)}^{\frac{2}{p}}\|g\|_{L^{2}(\Omega)}^{1-\frac{2}{p}}-\frac{(2 q-1)}{q^{2}}\|\nabla g\|_{L^{2}(\Omega)}^{2} \\
& \leq C\|f\|_{L^{2}(\Omega)}^{\frac{4}{p}}\|g\|_{L^{2}(\Omega)}^{2\left(1-\frac{2}{p}\right)}-\frac{(2 q-1)}{2 q^{2}}\|\nabla g\|_{L^{2}(\Omega)}^{2} \\
& \leq C_{q} \frac{(2 p-1)}{2 p^{2}}\|f\|_{L^{2}(\Omega)}^{2}+\frac{(2 q-1)}{4 q^{2}}\|g\|_{L^{2}(\Omega)}^{2}-\frac{(2 q-1)}{2 q^{2}}\|\nabla g\|_{L^{2}(\Omega)}^{2} \\
& \leq C_{q} \frac{(2 p-1)}{2 p^{2}}\|\nabla f\|_{L^{2}(\Omega)}^{2}-\frac{(2 q-1)}{4 q^{2}}\|\nabla g\|_{L^{2}(\Omega)}^{2},
\end{aligned}
$$


where we have used the Poincare inequality (38) in the last inequality.

Multiplying (39) by $C_{q}$ and adding the resulting equation to (40), we get

$$
\begin{aligned}
& \frac{d}{d t}\left(C_{q} \int_{\Omega} f^{2} r d r d z+\frac{1}{2 q} \int_{\Omega} g^{2} r d r d z\right) \\
& \leq C_{q}\left(C_{p}\|g\|_{L^{2}(\Omega)}^{\frac{1}{q}}\|\nabla f\|_{L^{2}(\Omega)}^{2}-\frac{(2 p-1)}{p^{2}}\|\nabla f\|_{L^{2}(\Omega)}^{2}\right) \\
& +C_{q} \frac{(2 p-1)}{2 p^{2}}\|\nabla f\|_{L^{2}(\Omega)}^{2}-\frac{(2 q-1)}{4 q^{2}}\|\nabla g\|_{L^{2}(\Omega)}^{2} \\
& \leq C_{q}\left(C_{p}\|g\|_{L^{2}(\Omega)}^{\frac{1}{q}}-\frac{(2 p-1)}{2 p^{2}}\right)\|\nabla f\|_{L^{2}(\Omega)}^{2} .
\end{aligned}
$$

Using (31), we have

$$
u_{1}(r, z, 0)=\epsilon^{\delta} U_{1}(\epsilon r, z), \quad \omega_{1}(r, z, 0)=\epsilon^{\delta} W_{1}(\epsilon r, z),
$$

where $U_{1}(\tilde{r}, \tilde{z})$ and $W_{1}(\tilde{r}, \tilde{z})$ have bounded $L^{2 p}$ and $L^{2 q}$ norms independent of $\epsilon$. We obtain

$$
\begin{aligned}
\left\|g_{0}\right\|_{L^{2}}^{\frac{1}{q}} & =\epsilon^{\delta-\frac{1}{q}}\left\|W_{1}\right\|_{L^{2 q}(\Omega)}, \\
\left\|f_{0}\right\|_{L^{2}}^{\frac{1}{p}} & =\epsilon^{\delta-\frac{1}{p}}\left\|U_{1}\right\|_{L^{2 p}(\Omega)} .
\end{aligned}
$$

We would like to choose $\epsilon$ small enough to ensure that the right hand side of (41) is negative. To this end, we require that

$$
C_{p}\|g\|_{L^{2}(\Omega)}^{\frac{1}{q}}(t) \leq \frac{(2 p-1)}{2 p^{2}}
$$

If (45) holds, we would get

$$
\frac{d}{d t}\left(\frac{C_{q}}{2 p} \int_{\Omega} f^{2} r d r d z+\frac{1}{2 q} \int_{\Omega} g^{2} r d r d z\right) \leq 0
$$

This implies that

$$
\frac{C_{q}}{2 p} \int_{\Omega} f^{2} r d r d z+\frac{1}{2 q} \int_{\Omega} g^{2} r d r d z \leq \frac{C_{q}}{2 p} \int_{\Omega} f_{0}^{2} r d r d z+\frac{1}{2 q} \int g_{0}^{2} r d r d z .
$$

This gives a global bound on $\left\|u_{1}\right\|_{L^{2 p}(\Omega)}$ and $\left\|\omega_{1}\right\|_{L^{2 q}(\Omega)}$ in terms of their initial data. In particular, we have by using (43)-(44) that

$$
\begin{aligned}
\|g\|_{L^{2}(\Omega)}^{\frac{1}{q}}(t) & \leq\left\|g_{0}\right\|_{L^{2}(\Omega)}^{\frac{1}{q}}+\left(C_{q} / 2\right)^{\frac{1}{2 q}}\left\|f_{0}\right\|_{L^{2}(\Omega)}^{\frac{2}{p}} \\
& \leq \epsilon^{\delta-\frac{1}{q}}\left\|W_{1}\right\|_{L^{2 q}(\Omega)}+\left(C_{q} / 2\right)^{\frac{1}{2 q}} \epsilon^{2 \delta-\frac{1}{q}}\left\|U_{1}\right\|_{L^{2 p}(\Omega)}^{2} .
\end{aligned}
$$


Using (48), we conclude that the condition (45) is satisfied if we choose $\epsilon$ to satisfy

$$
C_{p}\left(\epsilon^{\delta-\frac{1}{q}}\left\|W_{1}\right\|_{L^{2 q}(\Omega)}+\left(C_{q} / 2\right)^{\frac{1}{2 q}} \epsilon^{2 \delta-\frac{1}{q}}\left\|U_{1}\right\|_{L^{2 p}(\Omega)}^{2}\right) \leq \frac{(2 p-1)}{2 p^{2}}
$$

which is true by taking $q>1 / \delta$ and $\epsilon$ small enough. This condition would guarantee that the global estimate (47) is valid for all time. The global estimate (47) gives a global bound on $\left\|u_{1}\right\|_{L^{2 p}(\Omega)}$ and $\left\|\omega_{1}\right\|_{L^{2 q}(\Omega)}$. Using this global estimate, we can easily obtain a global bound on $\left\|u^{\theta}\right\|_{L^{2 p}}$ and $\left\|\omega^{\theta}\right\|_{L^{2 q}}$ over a bounded domain with $r \leq R$, which proves the global regularity of the 3D Navier-Stokes equations (see Remark 3). This completes the proof of Theorem 1.

Remark 4. The global regularity of the Navier-Stokes equations with initial data (3) follows almost exactly as the proof of Theorem 1 except that the bounds for $\left\|g_{0}\right\|_{L^{2}}$ and $\left\|f_{0}\right\|_{L^{2}}$ are different. By using the same rescaling, we just need to consider the rescaled initial data of the form

$$
u^{\theta}(r, z, 0)=U_{0}\left(\epsilon^{\delta} r, z\right), \quad \omega^{\theta}(r, z, 0)=W_{0}\left(\epsilon^{\delta} r, z\right)
$$

Due to the difference in the scaling, the bounds for $\left\|g_{0}\right\|_{L^{2 q}}$ and $\left\|f_{0}\right\|_{L^{2 q}}$ are now given by

$$
\begin{aligned}
& \left\|g_{0}\right\|_{L^{2}}^{\frac{1}{q}}=\epsilon^{\delta(1-1 / q)}\left\|W_{1}\right\|_{L^{2 q}(\Omega)}, \\
& \left\|f_{0}\right\|_{L^{2}}^{\frac{1}{p}}=\epsilon^{\delta(1-1 / p)}\left\|U_{1}\right\|_{L^{2 p}(\Omega)} .
\end{aligned}
$$

Thus the condition (45) is satisfied if we choose $q>1$ and $\epsilon$ small enough to satisfy

$$
C_{p}\left(\epsilon^{\delta\left(1-\frac{1}{q}\right)}\left\|W_{1}\right\|_{L^{2 q}(\Omega)}+\left(C_{q} / 2\right)^{\frac{1}{p}} \epsilon^{\delta\left(2-\frac{1}{q}\right)}\left\|U_{1}\right\|_{L^{2 p}(\Omega)}^{2}\right) \leq \frac{(2 p-1)}{2 p^{2}}
$$

Condition (53) will guarantee that the global estimate (47) is valid for all time.

Acknowledgments. We would like to thank Profs. Charles Fefferman and Fanghua Lin for their interests in this work and for some stimulating discussions. The research was in part supported by NSF under the NSF FRG grant DMS-0353838 and ITR Grant ACI-0204932.

\section{References}

[1] O. N. Boratav and R. B. Pelz, Direct numerical simulation of transition to turbulence from a high-symmetry initial condition, Phys. Fluids, 6 (1994), no. 8, 2757-2784.

[2] L. Caffarelli and R. Kohn and L. Nirenberg, Partial regularity of suitable weak solutions of the Navier-Stokes equations, Comm. Pure Appl. Math., 35, 771-831.

[3] D. Chae, Global regularity of the 2D Boussinesq equation with partial viscous terms, Advances in Math, 203 (2006), no. 2, 789-801. 
[4] D. Chae and J. Lee, On the regularity of the axisymmetric solutions of the Navier-Stokes equations, Math. Z., 239 (2002), no. 1, 645-671.

[5] C. C. Chen and R. M. Strain and T. P. Tsai and H. T. Yau, Lower bound on the blowup rate of axisymmetric Navier-Stokes equations, arXiv-preprint, math.AP/0701796v1, 2007.

[6] C. Fefferman, http://www.claymath.org/millenium/Navier-Stokes Equations.

[7] J. M. Greene and R. B. Pelz, Stability of postulated, self-similar, hydrodynamic blowup solutions, Phys. Rev. E, 62 (2000), no. 6, 7982-7986.

[8] T. Y. Hou and C. Li, Global well-posedness of the viscous Boussinesq equations, DCDS, 12 (2005), no. 1, 1-12.

[9] T. Y. Hou and C. Li, Dynamic stability of the 3D axi-symmetric Navier-Stokes equations with swirl, Comm. Pure Appl. Math., 61 (2008), no. 5, 661-697.

[10] T. Y. Hou and R. Li, Dynamic depletion of vortex stretching and non-blowup of the 3-D incompressible Euler equations, J. Nonlinear Science., 16 (2006), no. 6, 639-664.

[11] T. Y. Hou and R. Li, Computing nearly singular solutions using pseudo-spectral methods, J. Comput. Phys., 226 (2007), 379-397.

[12] T. Y. Hou and R. Li, Nonexistence of Local Self-Similar Blow-up for the 3D Incompressible Navier-Stokes Equations, Discrete and Continuous Dynamical Systems, 18 (2007), no. 4, 637-642.

[13] L. Escauriaza and G.G. Seregin and V. Sverak, $L_{3, \infty}$-solutions of Navier-Stokes equations and backward uniqueness, Uspekhi Mat. Nauk, 58 (2003), 3-44.

[14] R. M. Kerr, Evidence for a singularity of the three dimensional, incompressible Euler equations, Phys. Fluids, 5 (1993), no. 7, 1725-1746.

[15] R. M. Kerr, Velocity and scaling of collapsing Euler vortices, Phys. Fluids, 17 (2005), 075103-114.

[16] O.A. Ladyzhenskaya Mathematica problems of the dynamics of viscous incompressible fluids, Nauka, 1970, Moscow.

[17] F.H. Lin, A new proof of the Caffarelli-Korn-Nirenberg theorem, Comm. Pure Appl. Math., 51 (1998), no. 3, 241-257.

[18] J. G. Liu and W. C. Wang, Convergence Analysis of the Energy and Helicity Preserving Scheme for Axisymmetric Flows, SINUM, 44 (2006), no 6, 2456-2480.

[19] A. J. Majda and A. L. Bertozzi, Vorticity and Incompressible Flow, Cambridge University Press, 2002, Cambridge, UK. 
[20] J. Necas and M. Ruzicka and V. Sverak, On Leray's self-similar solutions of the NavierStokes equations, Acta Math., 176 (1996), 283-294.

[21] R. B. Pelz, Locally self-similar, finite-time collapse in a high-symmetry vortex filament model, Phys. Rev. E, 55 (1997), no. 2, 1617-1626.

[22] R. B. Pelz, Symmetry and hydrodynamic blow-up problem, J. Fluid Mech., 444 2001, 299-320.

[23] G. Raugel and G. Sell, Navier-Stokes equations on thin 3D domains I: Global attractors and global regularity of solutions, J. Amer. Math. Soc., 6 (1993), 503-568.

[24] G. Raugel and G. Sell, Navier-Stokes equations on thin 3D domains II: Global regularity of spatially periodic solutions, Nonlinear Partial Differential Equations and Their Applications, College de France Seminar, 205-247, Longman, XI (1994), Pitman Research Notes Math, Series 299.

[25] G. Raugel and G. Sell, Navier-Stokes equations on thin 3D domains III: Global and local attractors, Turbulence in Fluid Flows: A Dynamical Systems Approach, 137-163, Springer Verlag, 55 (1993), IMA Volumes in Mathematics and its Applications.

[26] E.M. Stein, Harmonic Analysis: Real-Variable Methods, Orthogonality, and Oscillatory Integrals, Princeton University Press, 1993, Princeton, New Jersey.

[27] R. Temam, Navier-Stokes Equations, American Mathematical Society, 2001, Providence, Rhode Island, second editio.

[28] T. P. Tsai, On Leray's self-similar solutions of the Navier-Stokes equations satisfying local energy estimates, Arch. Rational Mech. Anal., 143 (1998), 29-51. 